\section{A Ripple Effect in the Audiogram}

IN the course of some psycho-acoustic experiments it was found that one observer's thresholds of acuity at two frequencies separated by only a few tens of cycles differed by nearly 10 decibels. Dips in an audiogram are not uncommon; but the difference observed pointed to an uncommonly sharp change of sensitivity. Further tests revealed other equally sharp fluctuations in this observer's audiogram. Eventually a systematic exploration of the right-ear thresholds of this and one other observer was undertaken. Thresholds were measured at 10-cycle intervals over the range $400-3,000 \mathrm{c} . / \mathrm{s}$.

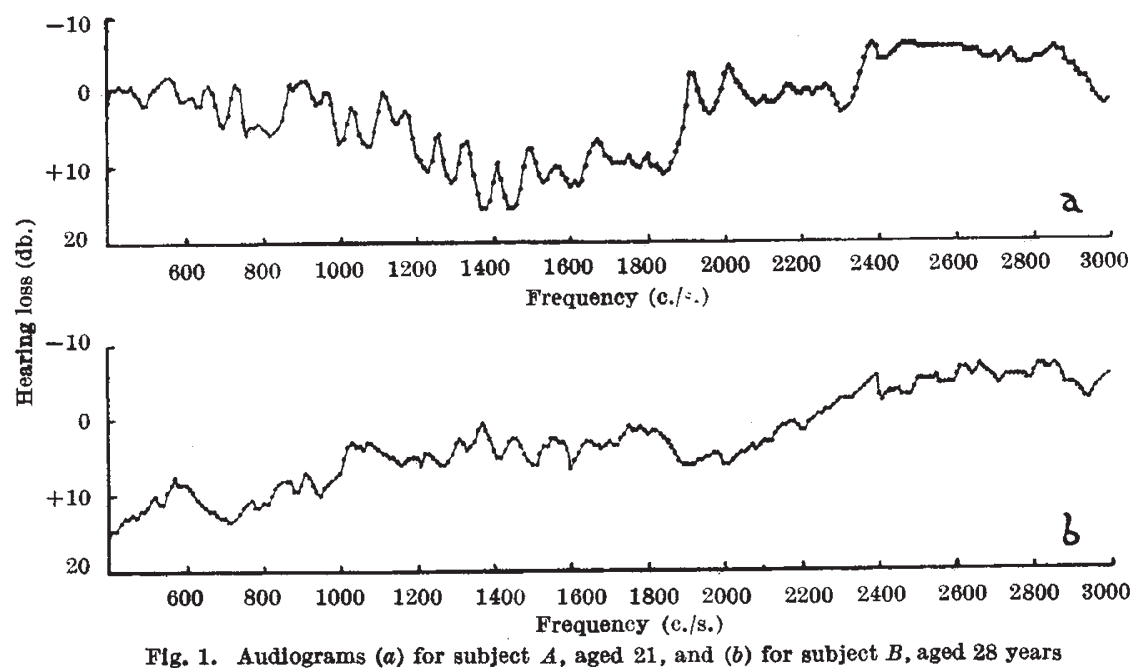
glare was required. the magnitude of the sensation of discomfort due to

An experiment was designed in which subjects, so far as possible naive in the context, were asked to adjust conditions in a room with glaring light sources until the sensation of discomfort was 'twice', 'ten times', 'one-half' and 'one-tenth' of the original level of discomfort. Eight out of twelve subjects were able to make judgments without trouble, the other four being either unco-operative or unable to follow what was required of them. The results were consistent and showed less variance than expected. The precise relations, which will be reported elsewhere, are not of importance in the present context because they depend on the experimental conditions.

It is of interest, however, that discomfort mag nitude was found not to be correlated linearly with (apparent) brightness of the glaring sources. Whereas, under the given conditions of adaptation, apparent brightness was known from previous work ${ }^{1}$ to be related to photometric brightness (luminance) by a power law of the form :

$$
M=k L^{0.5}
$$

where $M$ is apparent brightness magnitude and $L$ is luminance, the magnitude $G$ assigned to the glare discomfort was found,

The remarkable feature of these audiograms (Fig. 1) is the appearance of distinct ripples covering the range of frequencies up to about $2,000 \mathrm{c} / \mathrm{s}$. Further experiments showed (1) that the ripples were stable over a period of at least two months, (2) that the left-ear audiograms also showed ripples, but the periods and amplitudes differed, and (3) that the ripple effect must be a very common, if not a universal phenomenon, since three additional observers also had the same sort of rippling audiograms in the narrower band from 1,000 to $1,400 \mathrm{c} . / \mathrm{s}$. This ripple phenomenon seems to have been unnoticed hitherto.

Threshold sensitivity fluctuates quite widely from one test session to another, and even within a single session if it is at all prolonged. To demonstrate the ripple effect it was necessary, therefore, to keep the test sessions short ; but each session included measurements of threshold at frequency intervals which were small compared with the period of the ripple, and the frequency band covered was more than twice the period.

Admiralty Research

E. Euhiotr Laboratory, Teddington.
Feb. 6 .

\section{Magnitude of Discomfort caused by High Brightness}

Hrtherto the greatest care has been taken here in the studies of building efficiency to avoid methods of subjective judgment which depend on assessment of sensation magnitude in terms of numbers. For certain reasons, however, a numerical assessment of under essentially the same conditions, to follow a law

$$
G=k L^{1.28}
$$

In other words, whereas the luminance of the sources must be raised four times (antilog $\frac{\log _{10} 2}{0.5}$ ) to appear twice as bright, the sources will appear twice as uncomfortable (for glare) if their luminance is raised only 1.8 times (antilog $\frac{\log _{10} 2}{1 \cdot 23}$ ).

If these data are valid, it raises an important principle in practical engineering matters involving subjective judgments of magnitude, especially if this principle is applicable in other modalities, such as primary sensation (loudness or brightness) and also it does not follow that the magnitude of the secondary sensation is directly or simply linked to the magnitude of the primary sensation. Hence, if we wish to find out about discomfort or pain, we must assess discomfort or pain directly in relation to stimulus energy, and must not make implications from brightness or loudness magnitude assessments.

The experimental work was undertaken by $\mathbf{M r}$. R. C. Bradley (Cornell University) working at the Building Research Station with a grant from the Illuminating Engineering Research Institute of the United States.

Building Research Station,

Garston, Watford. Feb. 14 .

${ }^{1}$ Hopkinson, R. G., Nature, 178, 1065 (1956). noise. This is, that if a stimulus gives rise to both a an associated secondary sensation (pain or discomfort), 